\title{
Significant Correlation between Polymorphisms of UGT1A1 Gene and Low Irinotecan Toxicity in Colorectal Cancer Patients with FOLFIRI
}

\author{
Hsiang-Lin Tsai ${ }^{1,2,8}$, Chin-Fan Chen ${ }^{2}$, Chien-Yu Lu ${ }^{3}$, Wei-Yu Fang ${ }^{4}$, Deng-Chyang Wu ${ }^{3,5}$, I-Chen \\ $\mathrm{Wu}^{3}$, Maw-Chang Sheen ${ }^{2,5}$, Shiu-Ru Lin*,7,8 and Jaw-Yuan Wang ${ }^{*, 2,4,5,9}$ \\ ${ }^{I}$ Department of Emergency Medicine, Kaohsiung Municipal Hsiao-Kang Hospital, Kaohsiung Medical University, \\ Kaohsiung, Taiwan \\ ${ }^{2}$ Department of Surgery, Kaohsiung Medical University Hospital, Kaohsiung Medical University, Kaohsiung, Taiwan \\ ${ }^{3}$ Department of Internal Medicine, Kaohsiung Medical University Hospital, Kaohsiung Medical University, Kaohsiung, \\ Taiwan \\ ${ }^{4}$ Graduate Institute of Medical Genetics, College of Medicine, Kaohsiung Medical University, Kaohsiung, Taiwan \\ ${ }^{5}$ Faculty of Medicine, College of Medicine, Kaohsiung Medical University, Kaohsiung, Taiwan \\ ${ }^{6}$ Division of Colorectal Surgery, Department of Surgery, Taichung Veterans General Hospital, Taiwan \\ ${ }^{7}$ Department of Medical Research, Fooyin University Hospital, Pingtung, Taiwan \\ ${ }^{8}$ School of Medical and Health, Fooyin University, Kaohsiung, Taiwan \\ ${ }^{9}$ Graduate Institute of Medicine, College of Medicine, Kaohsiung Medical University, Kaohsiung, Taiwan
}

\begin{abstract}
Aim: To investigate the association between UDP-glucuronosyltransferase 1A1 (UGT1A1) genotypes and severe toxicity in Taiwanese patients with metastatic colorectal cancer (mCRC) receiving irinotecan chemotherapy.

Methods: We genotyped the UGT1A1 gene by direct sequencing. All the patients were evaluated to see whether the variant UGT1A1 genotype would correlate to severe toxicity of irinotecan consisting of grade III-IV neutropenia, diarrhea and nausea/vomiting. Genomic DNA was genotyped for UGT1A1, and patients were designated as 6/6, 6/7, or 7/7 depending on the number of TA repeats in the promoter region.

Results: The results showed that the genotype distribution of UGT1A1 in Taiwanese subjects differed significantly from that in Caucasians. Furthermore, patients with $6 / 7$ or $7 / 7$ genotype were associated with a higher incidence of grade III-IV neutropenia or diarrhea or nausea/vomiting (all $p<0.0001$ ). The less frequencies of $6 / 7$ and $7 / 7$ genotypes may be responsible for the considerably lower occurrence of grade III-IV neutropenia and diarrhea in Taiwanese patients. Indeed, the UGT1A1 genotype was closely related to clinical response $(\mathrm{p}=0.018)$.

Conclusion: UGT1A1 genotyping is a potential predictor of severe toxicity for Taiwanese mCRC patients treated with irinotecan chemotherapy, and may be useful to identify patients at-risk of toxicity, and thus could be used as a screening tool prior to therapy.
\end{abstract}

Key Words: UGT1A1, irinotecan, toxicity, metastatic colorectal cancer, FOLFIRI.

\section{INTRODUCTION}

Variation in the genetic constitution between individuals will have a major impact on the activation and metabolism of certain chemotherapeutic agents. Single-nucleotide polymorphisms (SNPs) account for over $90 \%$ of genetic variation in the human genome. Irinotecan, a semisynthetic camptothecin analog with topoisomerase I-inhibiting activity [1-3], shows excellent clinical efficacy in solid tumors such as lung, ovar-

*Address correspondence to these authors at the School of Medical and Health, Fooyin University, Kaohsiung, Taiwan and Department of Surgery, Kaohsiung Medical University Hospital, Kaohsiung Medical University, 100 Tzyou 1st Road, Kaohsiung 807, Taiwan; Tel: +886-7-3122805; Fax: +886-7-3114679; E-mail: cy614112@ms14.hinet.net

Shiu-Ru Lin and Jaw-Yuan Wang contributed equally to this paper. ian, and colorectal cancers (CRC) [4-6]. Irinotecan is a prodrug that is converted by carboxylesterase to an active metabolite 7-ethyl-10-hydroxycamptothecin ( $\mathrm{SN}-38$ ), which is 100 - to 1,000 -fold more cytotoxic than irinotecan. SN-38 can be inactivated through glucuronidation by a member of the uridine diphosphate glucuronosyltransferase (UGTs) family in the liver to an inactive metabolite, SN-38 glucuronide $(\mathrm{SN}-38 \mathrm{G})[2,3]$. Therefore, the conversion of SN-38 to SN$38 \mathrm{G}$ by hepatic UGTs is a critical step in the sequential metabolic pathway of irinotecan. Because glucuronidation is the major route of detoxification and elimination of active metabolite SN-38, inherited differences in irinotecan glucuronidation capacity may have an important influence on the pharmacokinetics and toxicity of this drug $[7,8]$. 
A dinucleotide repeat in a TATA box in the UGTIAl promoter results in altered UGT1A1 activity [9]. Reduced UGT1A1 is linked to a high risk (approximately four-fold) of severe toxicity from irinotecan treatment, including doselimiting diarrhea and neutropenia [10]. The variable number of TA repeats ranges from five to eight copies, six TA repeats (6TA/6TA) represent the most common allele, with up to $33 \%$ in Caucasians having a variant allele containing seven repeats $(U G T 1 A 1 * 28)$ [11]. The clinical significance between patients with the UGTIAI $* 28$ allele and reduced UGT1A1 expression, and consequently reduced SN38 glucuronidation and irinotecan-related toxicity is well established [11-14]. Assessment of the presence of the UGTIAI $* 28$ allele in cancer patients prior to administration of irinotecan may predict individuals at risk for severe toxicity from irinotecan, allowing the selection of lower doses or alternative therapies.

Several recent studies have suggested that a statistically significant relationship is found between the appearance of severe diarrhea or/and hematological toxicities and the homozygote $U G T 1 A 1 * 28$ genotype when compared with UGT1Al wild-type Caucasian CRC patients [15-18]; however, no information in Taiwanese patients with metastatic CRC (mCRC) treated with irinotecan-based chemotherapy has ever been reported. The aim of our study was to assess the role of UGTIAI gene polymorphisms on the occurrence of severe toxicities and response in Taiwanese patients with mCRC receiving FOLFIRI regimen. Furthermore, we have also compared the UGTIAl gene polymorphisms and incidence of toxicities of mCRC in Asian and Caucasian patients receiving FOLFIRI regimen.

\section{MATERIALS AND METHODOLOGY}

\section{Patients and Treatment}

This prospective pilot study was conducted from January 2005 to March 2008, including 72 cytologically or histologically confirmed mCRC (International Union Against Cancer (UICC) stage IV) patients, and performance status (PS) $\leqq 2$ on the Eastern Cooperative Oncology Group (ECOG) scale, in the Kaohsiung Medical University Hospital receiving FOLFIRI regimen. Written informed consent was obtained from all subjects and/or guardians for the use of their blood samples. Sample acquisition and subsequent use were also approved by the institutional review board of the Kaohsiung Medical University Hospital. The FOLFIRI regimen is as follows: irinotecan $\left(180 \mathrm{mg} / \mathrm{m}^{2}\right)$ on day 1 with Leucovorin (LV) $\left(200 \mathrm{mg} / \mathrm{m}^{2}\right)$ administered as a 2-hour infusion before 5 -fluorouracil (5-FU) $\left(400 \mathrm{mg} / \mathrm{m}^{2}\right)$ administered as an intravenous bolus injection, and 5 -FU $\left(2400 \mathrm{mg} / \mathrm{m}^{2}\right)$ as a 46 -hour infusion immediately after 5 -FU bolus injection on days 1 and 2. Courses were repeated every two weeks in the presence of an absolute neutrophil count $\geq 1500 / \mu 1$ and platelet count $\geq 100,000 / \mu 1$, and recovery of any extra-hematological toxicity; otherwise, treatment was postponed for one or two weeks until recovery. Also, the chemotherapy was continued until the disease progressed or unacceptable toxicities developed or the patient refused further treatment with FOLFIRI. Any grade III or IV adverse events resulted in an approximately $20 \%$ dose reduction of irinotecan for subsequent cycles. Persistent grade II or worse adverse events delayed therapy until recovery. The use of colony-stimulating factors was allowed if medically justified. Intensive treatment with loperamide, if needed, was used for diarrhea. All patients were included in safety and efficacy analyses. Safety assessment and laboratory tests were performed biweekly. The severity of adverse effects was evaluated according to the National Cancer Institute Common Toxicity Criteria (NCICTC), version 2.0 (http://ctep.cancer.gov/reporting/ctc.html; accessed in September 2008). Baseline measurement of tumor size was based on computed tomography (CT) scan or $\mathrm{X}$-ray or other radiological means. Tumors were measured at 6- to 8-week intervals, and objective response was evaluated according to the response evaluation criteria for solid tumors (RECIST) [19], as well as the best response being recorded. For the analysis, patients with a complete or partial response were grouped in responders; patients with a stable disease or progressive disease were grouped in non-responders.

\section{Genotyping}

Constitutional gene polymorphisms were analyzed by DNA extraction from $4 \mathrm{~mL}$ peripheral blood using PUREGENE $^{\mathbb{B}}$ DNA Isolation Kit (Gentra Systems Inc., Minneapolis, MN, USA). All genomic DNA from the patients were analyzed using direct sequencing technique for the determination of genotypes of UGTIAl promoter region. The primers used in this study were designed by using primer 3 free software (http://web.umassmed.edu/bioapps/primer3_www. cgi; accessed in May 2008). The sequences of the forward and reverse primers were 5'- AGTCACGTGACACAGTCAAACA-3' and CTTTGCTCCTGCCAGAGGTT-3', respectively. The PCR reaction volume was $40 \mu \mathrm{l}$ and the PCR conditions for these polymorphisms were described as follows: GSTP1: $94^{\circ} \mathrm{C}$ for $5 \mathrm{~min} ; 30$ cycles of denaturation for $30 \mathrm{sec}$ at $94^{\circ} \mathrm{C}$, annealing for $20 \mathrm{sec}$ at $67.5^{\circ} \mathrm{C}$, primer extension for $20 \mathrm{sec}$ at $72^{\circ} \mathrm{C}$, and a final extension for $10 \mathrm{~min}$ at $72^{\circ} \mathrm{C}$. Genotype verifications were carried out by fragment analysis of the PCR product using the automated capillary electrophoresis on the ABI PRISM 310 Genetic Analyzer (Applied Biosystems, Foster City, Calif., USA) and analyzed with GeneScan and Genotyper software (Applied Biosystems).

\section{Statistical Analysis}

All data were analyzed using Statistical Package for the Social Sciences Version 12.0 software (SPSS Inc., Chicago, Ill, USA). All genotypes were tested whether they were distributed according to the Hardy-Weinberg equilibrium or not. The Hardy-Weinberg equilibrium assumption was assessed by the standard method of matching the observed numbers of individuals in the different genotype categories with those expected under Hardy-Weinberg equilibrium for the estimated allele frequency. $\chi^{2}$ test for deviation from Hardy-Weinberg equilibrium was used to estimate differences in allele frequencies. Differences between categorical variables were measured by the two-sided Pearson $\chi^{2}$ test and Fisher's exact test. A $P$ value less than 0.05 was considered statistically significant.

\section{RESULTS}

\section{Characteristics of Patients}

Of these 72 mCRC patients, 43 were males and 29 were females. The median age was 60 years (range, 20-80). Fortyfive tumors were located in the colon, and 27 tumors were in 
the rectum. Two (2.8\%), 19 (26.4\%), 24 (33.3\%), and 27 (37.5\%) patients were subsequently categorized into complete response, partial response, stable disease, and progressive disease, respectively, according to RECIST. Consequently, $21(29.2 \%)$ and $51(70.8 \%)$ patients were finally classified into responders and non-responders. Patients' characteristics are depicted in Table $\mathbf{1 .}$

Table 1. Baseline Characteristics of 72 Metastatic Colorectal Cancer Patients Undergoing Irinotecan-Based Chemotherapy

\begin{tabular}{|c|c|}
\hline Variables & No. $(n=72)$ \\
\hline \multicolumn{2}{|l|}{ Gender } \\
\hline Male & $43(59.7)$ \\
\hline Female & $29(40.3)$ \\
\hline Median age (range, year) & $60(24-80)$ \\
\hline \multicolumn{2}{|c|}{ Performance status (ECOG) } \\
\hline 0 & $41(56.9)$ \\
\hline 1 & $26(36.1)$ \\
\hline 2 & $5(7)$ \\
\hline \multicolumn{2}{|l|}{ Tumor location } \\
\hline Colon & $45(62.5)$ \\
\hline Rectum & $27(37.5)$ \\
\hline \multicolumn{2}{|l|}{ As nth-line chemotherapy } \\
\hline 1 & $34(47.2)$ \\
\hline 2 & $34(47.2)$ \\
\hline 3 & $4(5.6)$ \\
\hline \multicolumn{2}{|l|}{ Sites of metastases } \\
\hline Liver & $42(58.3)$ \\
\hline Lung & $21(29.2)$ \\
\hline Local recurrence & 17 (23.6) \\
\hline Peritoneum & $12(16.7)$ \\
\hline Bone & $7(9.7)$ \\
\hline Distant lymph nodes & $5(7)$ \\
\hline Others & $8(11.1)$ \\
\hline Multiple & $28(38.9)$ \\
\hline \multicolumn{2}{|l|}{ Objective response } \\
\hline Complete response & $2(2.8)$ \\
\hline Partial response & $19(26.4)$ \\
\hline Stable disease & $24(33.3)$ \\
\hline Progressive disease & $27(37.5)$ \\
\hline
\end{tabular}

Values in parenthesis are percentages. ECOG, Eastern Cooperative Oncology Group.

\section{UGT1A1 Genotype with Toxicity and Clinical Response Among Various Races}

The frequencies of 6TA/6TA, 6TA/7TA, and 7TA/7TA genotypes were $53(73.6 \%), 18(25 \%)$, and $1(1.4 \%)$ of 72 patients respectively. There was a marked relationship between the appearance of severe (Grade III/IV) neutropenia, diarrhea, and nausea/vomiting and the heterozygous (6TA/7TA) or homozygous $U G T 1 A 1 * 28$ (7TA/7TA) conditions (all $\mathrm{p}<0.0001$, Table 2). Of these patients with severe toxicities, $87.5 \%$ to $100 \%$ were heterozygous and homozygous $U G T 1 A 1 * 28$ conditions; while in patients without severe toxicities only $18.7 \%$ to $20.1 \%$ were heterozygous $U G T 1 A 1 * 28$ genotype. Table $\mathbf{3}$ summarized the genotype and allele frequencies of UGTIA1 among our study and other previous studies $[15,17,20,21]$. A significant lower frequency of 6TA/7TA and 7TA/7TA genotypes in Taiwanese patients when compared to that of Caucasian patients was observed ( $p<0.0001)$. However, no statistical difference of the frequency of 6TA/7TA and 7TA/7TA between our study and other Asian or Japanese studies was found. Less than one-fifth of Asian patients were 7TA allele, of which the frequency of allele distribution was significantly lower than Caucasian patients $(\mathrm{p}<0.0001)$. A considerably higher incidence of severe neutropenia $(\mathrm{p}=0.011)$ and diarrhea $(\mathrm{p}<0.0001)$ in Caucasian patients than in Taiwanese patients was noted, though a significant difference was not achieved in severe nausea/vomiting $(p=0.224$; Table 4). Furthermore, a prominent correlation between the UGT1A1 genotypes and clinical response to FOLFIRI chemotherapy was shown $(\mathrm{p}=0.018$, Table 5). Ten of $21(47.6 \%)$ responders were 6TA/7TA or 7TA/TA genotypes, whereas 9 of $51(17.6 \%)$ non-responders were 6TA/7TA or 7TA/TA genotypes.

\section{DISCUSSION}

Due to its efficacy, irinotecan is currently approved as first-line therapy in mCRC treatment, and can cause severe unpredictable gastrointestinal and hematologic toxicity. Much of the inter-individual variability in drug toxicities is attributable to the presence of SNPs in genes encoding drugmetabolizing enzymes and drug transporters involved in the biochemical pathway of irinotecan. Although more than 50 genetic lesions in the UGT1Al gene have been described [22], the UGTIA1*28 allele (the most frequent polymorphism in Caucasian populations) plays a vital role in the development of toxicity after irinotecan chemotherapy. To elucidate the most important functional polymorphism that determines the severe toxicities of Taiwanese patients with mCRC treated with irinotecan-based chemotherapy, we examined the polymorphisms of the UGTIAl genes involved in irinotecan-metabolizing enzymes. The present investigation shows that there is a significant difference in the genotype and allele distribution between Taiwanese and Caucasian mCRC patients. Likewise, several recently published studies have shown the disposition of irinotecan and its metabolites to differ among different ethnic groups $[14,18,23,24]$. When compared with other ethnic groups, the distribution of the wild-type 6TA/6TA genotype or 6TA allele in Taiwanese mCRC patients was relatively high (approximately two-fold) and similar to the Japanese and other Asian populations, but different from Caucasian patients. Notably, the 6TA allele and 7TA allele frequencies in Caucasian and Asian (including Taiwan) populations are different.

The development of severe toxicities including neutropenia, diarrhea, and nausea/vomiting is closely associated with the 6TA/7TA and 7TA/7TA genotypes in our patients treated with FOLFIRI regimen. Several recent studies have 
Table 2. Association Between UGT1A1 Polymorphism and the Occurrence of NCI-CTI Grade III and IV Toxicities

\begin{tabular}{|c|c|c|c|c|c|c|c|}
\hline \multirow[t]{2}{*}{ Genotype } & \multirow{2}{*}{$\begin{array}{c}\text { No. } \\
(n=72)\end{array}$} & \multicolumn{2}{|c|}{ Grade III/IV Neutropenia } & \multicolumn{2}{|c|}{ Grade III/IV Diarrhea } & \multicolumn{2}{|c|}{ Grade III/IV Nausea/Vomiting } \\
\hline & & $\begin{array}{c}\text { Experienced } \\
\text { No. }(n=6)\end{array}$ & $\begin{array}{l}\text { Not Experienced } \\
\qquad(n=66)\end{array}$ & $\begin{array}{c}\text { Experienced } \\
\text { No. }(n=4)\end{array}$ & $\begin{array}{l}\text { Not Experienced } \\
\text { No. }(n=68)\end{array}$ & $\begin{array}{c}\text { Experienced } \\
\text { No. }(n=8)\end{array}$ & $\begin{array}{c}\text { Not Experienced } \\
\text { No. }(n=64)\end{array}$ \\
\hline $6 / 6$ & $53(73.6)$ & $0(0)$ & $53(80.3)$ & $0(0)$ & $53(77.9)$ & $1(12.5)$ & $52(81.3)$ \\
\hline$P$ & & $<0.0001$ & & $<0.0001$ & & $<0.0001$ & \\
\hline
\end{tabular}

Values in parentheses are percentages. NCI-CTI, National Cancer Institute-Common Toxicity Criteria; UGT1A1, uridine diphosphate glucuronosyl transferase.

Table 3. Comparison of Distribution of UGT1A1genotypes Between our Taiwanese Colorectal Cancer Patients and other Populations

\begin{tabular}{|c|c|c|c|c|c|}
\hline & $\begin{array}{c}\text { Caucasian } \\
\text { No. }(\boldsymbol{n}=\mathbf{9 5})\end{array}$ & $\begin{array}{c}\text { Caucasian } \\
\text { No. }(\boldsymbol{n}=\mathbf{7 2})\end{array}$ & $\begin{array}{c}\text { Japanese } \\
\text { No. }(\boldsymbol{n}=\mathbf{1 1 8})\end{array}$ & $\begin{array}{c}\text { Asian } \\
\text { No. }(\boldsymbol{n}=\mathbf{4 5})\end{array}$ & \\
\hline \hline Nenotype $(\boldsymbol{n}=\mathbf{7 2})$
\end{tabular}

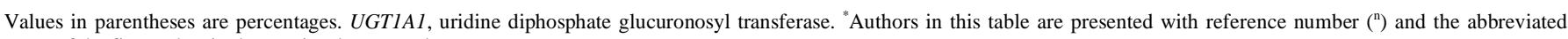
name of the first author in the previously reported paper.

Table 4. Comparison of Association Between UGT1A1 Polymorphism and the Occurrence of NCI-CTI Grade III and IV Toxicities Between our Taiwanese Colorectal Cancer Patients and Caucasians (Marcuello [15])

\begin{tabular}{|c|c|c|c|c|c|c|}
\hline Genotype & \multicolumn{2}{|c|}{ Grade III-IV Neutropenia } & \multicolumn{2}{c|}{ Grade III-IV Diarrhea } & \multicolumn{2}{c|}{ Grade III-IV Nausea/Vomiting } \\
\hline & Caucasian No. & Taiwanese No. & Caucasian No. & Taiwanese No. & Caucasian No. & Taiwanese No. \\
\hline \hline $6 / 6$ & $6(15)^{\dagger}$ & $0(0)^{\dagger}$ & $7(17)^{\dagger}$ & $0(0)^{\dagger}$ & $5(12)^{\dagger}$ & $1(1.9)^{\dagger}$ \\
\hline $6 / 7$ & $12(27)^{\dagger}$ & $5(27.8)^{\dagger}$ & $15(33)^{\dagger}$ & $3(16.7)^{\dagger}$ & $10(22)^{\dagger}$ & $6(33.3)^{\dagger}$ \\
\hline $7 / 7$ & $4(40)^{\dagger}$ & $1(100)^{\dagger}$ & $7(70)^{\dagger}$ & $1(100)^{\dagger}$ & $2(20)^{\dagger}$ & $1(100)^{\dagger}$ \\
\hline Overall & $22(23.2)^{\dagger}$ & $6(8.3)^{\dagger}$ & $29(30.5)^{\dagger}$ & $4(5.6)^{\dagger}$ & $17(17.9)^{\dagger}$ & $8(11.1)^{\dagger}$ \\
\hline$P^{\ddagger}$ & 0.011 & - & $<0.0001$ & - & 0.224 \\
\hline
\end{tabular}

Values in parentheses are percentages of all genotyping patients. NCI-CTI, National Cancer Institute-Common Toxicity Criteria. ${ }^{*}$ Authors in this table are presented with reference number $\left({ }^{\mathrm{n}}\right)$ and the abbreviated name of the first author in the previously reported paper. ${ }^{\dagger}$ Indicates the percentage of all patients with the same genotype. ${ }^{*}$ Comparison between the overall incidences of grade III and IV toxicities. 
Table 5. Correlation Between UGT1A1 Polymorphism and Response to Chemotherapy

\begin{tabular}{|c|c|c|c|}
\hline & $\begin{array}{c}\text { Responder }^{*} \\
(\boldsymbol{n}=\mathbf{2 1})\end{array}$ & $\begin{array}{c}\text { Nonresponder }^{\dagger} \\
(\boldsymbol{n}=\mathbf{5 1})\end{array}$ & $\boldsymbol{P}$ \\
\hline \hline Genotype & & $42(82.4)$ & 0.018 \\
\hline $6 / 6$ & $11(52.4)$ & $9(17.6)$ & - \\
\hline $6 / 7$ & $9(42.9)$ & $0(0)$ & - \\
\hline $7 / 7$ & $1(4.7)$ & & \\
\hline
\end{tabular}

Values in parentheses are percentages. UGT1A1, uridine diphosphate glucuronosyl transferase, ${ }^{*}$ responder, complete response and partial response; ${ }^{\dagger}$ nonresponder, stable disease and progressive disease.

evaluated the impact of the UGT1A1 polymorphism on the main dose-limiting toxicities of irinotecan (i.e., diarrhea and neutropenia). For example, consistent with our observation, Côté and his colleagues showed that CRC patients with $U G T 1 A 1 * 28$ polymorphism had more frequent severe hematologic toxicity $(50 \%)$ than patients homozygous for wildtype allele (12.5\%) [16]. Meanwhile, Toffoli et al. indicated that $U G T 1 A 1 * 28$ polymorphism was associated with a higher risk of grade III to IV hematologic toxicity (odds ratio, 8.63 ; $95 \%$ CI, 1.31 to 56.55 ), which was only relevant for the first cycle of irinotecan chemotherapy. ${ }^{18}$ Regarding the diarrhea, de Jong et al. also found that the presence of at least one $U G T 1 A 1 * 28$ allele was strongly related to the incidence of grade II-III diarrhea [25]. Ando et al. stressed that the 7TA/7TA and 6TA/7TA genotypes would be a significant risk factor for severe irinotecan toxicity [20]. Moreover, Araki et al. demonstrated that Japanese cancer patients who were heterozygous and homozygous $U G T 1 A 1 * 28$ genotypes had markedly lower SN-38 glucuronidation activity than those who were patients homozygous for wild-type, potentially increasing susceptibility to toxicity of irinotecan [26]. However, the association between UGT1Al polymorphism and development of adverse event is not always identical in all studies. In a recent prospective study in which irinotecan was the sole chemotherapeutic agent, a significant association was found between the UGT1A1 genotype and neutropenia but not with diarrhea [27]; whereas in another retrospective study in which a combined irinotecan regimen was used, diarrhea but not neutropenia was found to be associated significantly with the UGT1A1 promoter polymorphism [15]. The different irinotecan dosing schedules and combination chemotherapy regimen would probably lead to their impact on the various degrees and severity of drug toxicities, as well as the subsequent analyzing results.

Furthermore, the frequency of neutropenia and diarrhea in Caucasian patients is significantly higher (approximately three- to five-fold) than that in our patients, despite the frequency of nausea/vomiting being not statistically different. Prevalence of the 7TA/7TA genotype is very high in Caucasians (10\% of the population) and in African people (20$25 \%$ ) $[15,28]$; conversely, in Asian populations [20, 21, 26], the 7TA/7TA genotype is quite rare, a finding that is in keeping with the results of the current study. From the above findings, we suggest that the relatively lesser presence of 6TA/7TA and 7TA/7TA genotypes may probably play a crucial role in the lower frequency of irinotecan-induced toxicities encountered in Taiwanese mCRC patients. Otherwise, in the current study, the heterozygous or homozygous
$U G T 1 A 1 * 28$ seemed to be associated with increased clinical benefit and tumor response. Toffoli et al. also pointed out that heterozygous 6TA/7TA and homozygous 7TA/7TA mCRC patients had a significantly reduced risk of stable diseases or progressive diseases compared with the wild-type genotype [18].

Due to the limited case numbers from these studies and variable pharmacodynamics of irinotecan, the available data can only really provide for an estimate of adverse risk effects in patient subpopulations rather than any predictable riskbenefit for an individual patient undergoing treatment (http://www.cdc.gov/genomics/gtesting/EGAPP/about.htm; accessed in May 2009). Therefore, larger mCRC cases to definitely establish the clinical relevance of UGTIAl gene polymorphisms on the occurrence of severe toxicities are mandatory. Another important issue is that such geneticallybased reduction in irinotecan dosage may result in diminished tumor responsiveness and cancer-specific outcomes since there are significantly higher response rates to standard irinotecan dosing in cases where the phenotype would predict for the highest rate of adverse events [18, 29]. Hence, a careful consideration before irinotecan dose reduction in patients carrying the polymorphic $\mathrm{TA}_{7}$ allele is recommended. The only way to resolve this issue is to conduct a prospective randomized controlled trial which examines both reduced irinotecan dosage in patients with CRC based on their UGT1Al genotype as well as the opposite side of the coin of dose escalation amongst wild-types individuals to improve tumor responsiveness with minimal effects on adverse drug events.

\section{CONCLUSION}

Taiwanese mCRC patients with heterozygous or homozygous $U G T 1 A 1 * 28$ have a higher incidence of irinotecanrelated severe toxicities. Therefore, determination of the UGT1A1 genotypes may be potentially beneficial for identifying our patients at-risk of developing a severe or potentially life-threatening toxicity after FOLFIRI regimen.

\section{ACKNOWLEDGEMENTS}

The authors would like to thank Yang Yi-Sing (Statistical Analysis Laboratory, Department of Medical Research, Kaohsiung Medical University Hospital) for her assistance of the statistical analysis. We also thank Drs. Jan-Sing Hsieh, Yu-Chung Su, Che-Jen Huang, Yong-Sang Pan, Jeng-Yih $\mathrm{Wu}$, and Hon-Man Chan for having contributed materially to the paper. 


\section{REFERENCES}

[1] Jaxel C, Kohn KW, Wani MC, Wall ME, Pommier Y. Structureactivity study of the actions of camptothecin derivatives on mammalian topoisomerase. I: Evidence for a specific receptor site and relation to antitumor activity. Cancer Res 1989; 49: 1465-9.

[2] Mathijssen RH, van Alphen RJ, Verweij J, Loos WJ, Nooter K, Stoter G, Sparreboom A. Clinical pharmacokinetics and metabolism of irinotecan (CPT-11). Clin Cancer Res 2001; 7: 2182-94.

[3] Zamboni WC, Stewart CF, Cheshire PJ, et al. Studies of the efficacy and pharmacology of irinotecan against human colon tumor xenograft models. Clin Cancer Res 1998; 4: 743-53.

[4] Negoro S, Masuda N, Takada Y, et al. Randomised phase III trial of irinotecan combined with cisplatin for advanced non-small-cell lung cancer. Br J Cancer 2003; 88: 335-41.

[5] Bodurka DC, Levenback C, Wolf JK, et al. Phase II trial of irinotecan in patients with metastatic epithelial ovarian cancer or peritoneal cancer. J Clin Oncol 2003; 21: 291-7.

[6] Saltz LB, Cox JV, Blanke C, et al. Irinotecan plus fluorouracil and leucovorin for metastatic colorectal cancer: Irinotecan Study Group. N Engl J Med 2000; 343: 905-14.

[7] Gupta E, Mick R, Ramirez J, et al. Pharmacokinetic and pharmacodynamic evaluation of the topoisomerase inhibitor irinotecan in cancer patients. J Clin Oncol 1997; 15: 1502-10.

[8] Iyer L, King CD, Whitington PF, et al. Genetic predisposition to the metabolism of irinotecan (CPT-11). Role of uridine diphosphate glucuronosyltransferase isoform 1A1 in the glucuronidation of its active metabolite (SN-38) in human liver microsomes. J Clin Invest 1998; 101: 847-54.

[9] Monaghan G, Ryan M, Seddon R, Hume R, Burchell B. Genetic variation in bilirubin UPD-glucuronosyltransferase gene promoter and Gilbert's syndrome. Lancet 1996; 347: 578-81.

[10] Wasserman E, Myara A, Lokiec F, et al. Severe CPT-11 toxicity in patients with Gilbert's syndrome: two case reports. Ann Oncol 1997; 8: 1049-51

[11] Iyer L, Hall D, Das S, et al. Phenotype-genotype correlation of in vitro $\mathrm{SN}-38$ (active metabolite of irinotecan) and bilirubin glucuronidation in human liver tissue with UGT1A1 promoter polymorphism. Clin Pharmacol Ther 1999; 65: 576-82.

[12] Ando Y, Saka H, Asai G, Sugiura S, Shimokata K, Kamataki T. UGT1A1 genotypes and glucuronidation of SN-38, the active metabolite of irinotecan. Ann Oncol 1998; 9: 845-7.

[13] Fisher MB, Vandenbranden M, Findlay K, et al. Tissue distribution and interindividual variation in human UDPglucuronosyltransferase activity: Relationship between UGT1A1 promoter genotype and variability in a liver bank. Pharmacogenetics 2000; 10: 727-39.

[14] Iyer L, Das S, Janisch L, et al. UGT1A1*28 polymorphism as a determinant of irinotecan disposition and toxicity. Pharmacogenomics J 2002; 2: 43-7.

[15] Marcuello E, Altés A, Menoyo A, Del Rio E, Gómez-Pardo M, Baiget M. UGT1A1 gene variations and irinotecan treatment in patients with metastatic colorectal cancer. Br J Cancer 2004; 91: 67882.
[16] Côté JF, Kirzin S, Kramar A, et al. UGT1A1 polymorphism can predict hematologic toxicity in patients treated with irinotecan. Clin Cancer Res 2007; 13: 3269-75.

[17] Rouits E, Boisdron-Celle M, Dumont A, Guérin O, Morel A, Gamelin E. Relevance of different UGT1A1 polymorphisms in irinotecan-induced toxicity: a molecular and clinical study of 75 patients. Clin Cancer Res 2004; 10: 5151-9.

[18] Toffoli G, Cecchin E, Corona G, et al. The role of UGT1A1*28 polymorphism in the pharmacodynamics and pharmacokinetics of irinotecan in patients with metastatic colorectal cancer. J Clin Oncol 2006; 24: 3061-8.

[19] Therasse P, Arbuck SG, Eisenhauer EA, et al. New guidelines to evaluate the response to treatment in solid tumors. European Organization for Research and Treatment of Cancer, National Cance Institute of the United States, National Cancer Institute of Canada. J Natl Cancer Inst 2000; 92: 205-16.

[20] Ando Y, Saka H, Ando M, et al. Polymorphisms of UDPglucuronosyltransferase gene and irinotecan toxicity: a pharmacogenetic analysis. Cancer Res 2000; 60: 6921-6.

[21] Jada SR, Lim R, Wong CI, et al. Role of UGT1A1*6, UGT1A1*28 and $\mathrm{ABCG} 2 \mathrm{c} .421 \mathrm{C}>\mathrm{A}$ polymorphisms in irinotecan-induced neutropenia in Asian cancer patients. Cancer Sci 2007; 98: 1461-7.

[22] Kadakol A, Ghosh SS, Sappal BS, Sharma G, Chowdhury JR, Chowdhury NR. Genetic lesions of bilirubin uridinediphosphoglucuronate glucuronosyl-transferase (UGT1A1) causing Crigler-Najjar and Gilbert's syndromes: correlation of genotype to phenotype. Hum Mutat 2000; 16: 297-306.

[23] Chowbay B, Zhou S, Lee EJ. An interethnic comparison of polymorphisms of the genes encoding drug-metabolizing enzymes an drug transporters: experience in Singapore. Drug Metab Rev 2005; 37: 327-78.

[24] Han JY, Lim HS, Shin ES, Yoo YK, Park YH, Lee JE. Comprehensive analysis of UGT1A polymorphisms predictive for pharmacokinetics and treatment outcome in patients with non-small-cell lung cancer treated with irinotecan and cisplatin. J Clin Oncol 2006; 24: 2237-44.

[25] de Jong FA, Kehrer DF, Mathijssen RH, et al. Prophylaxis of irinotecan-induced diarrhea with neomycin and potential role for UGT1A1*28 genotype screening: a double-blind, randomized, placebo-controlled study. Oncologist 2006; 11: 944-54

[26] Araki K, Fujita K, Ando Y, et al. Pharmacogenetic impact of polymorphisms in the coding region of the UGT1A1 gene on SN-38 glucuronidation in Japanese patients with cancer. Cancer Sci 2006 97: $1255-9$

[27] Innocenti F, Undevia SD, Iyer L, et al. Genetic variants in the UDP-glucuronosyltransferase $1 \mathrm{~A} 1$ gene predict the risk of severe neutropenia of irinotecan. J Clin Oncol 2004; 22: 1382-8.

[28] Beutler E, Gelbart T, Demina A. Racial variability in the UDPglucuronosyltransferase 1 (UGT1A1) promoter: a balanced polymorphism for regulation of bilirubin metabolism? Proc Natl Acad Sci USA 1998; 95: 8170-4.

[29] Palomaki GE, Bradley LA, Douglas MP, Kolor K, Dotson WD. Can UGT1A1 genotyping reduce morbidity and mortality in patients with metastatic colorectal cancer treated with irinotecan? An evidence-based review. Genet Med 2009; 11: 21-34. 